\title{
Rigidity and the Lower Bound Theorem for Doubly Cohen-Macaulay Complexes
}

\author{
Eran Nevo
}

Received: 10 August 2005 / Revised: 11 January 2006 /

Published online: 5 September 2007

(C) Springer Science+Business Media, LLC 2007

\begin{abstract}
We prove that for $d \geq 3$, the 1-skeleton of any $(d-1)$-dimensional doubly Cohen-Macaulay (abbreviated 2-CM) complex is generically $d$-rigid. This implies that Barnette's lower bound inequalities for boundary complexes of simplicial polytopes (Barnette, D. Isr. J. Math. 10:121-125, 1971; Barnette, D. Pac. J. Math. 46:349-354, 1973) hold for every 2-CM complex of dimension $\geq 2$ (see Kalai, G. Invent. Math. 88:125-151, 1987). Moreover, the initial part $\left(g_{0}, g_{1}, g_{2}\right)$ of the $g$-vector of a 2-CM complex (of dimension $\geq 3$ ) is an $M$-sequence. It was conjectured by Björner and Swartz (J. Comb. Theory Ser. A 113:1305-1320, 2006) that the entire $g$-vector of a 2-CM complex is an $M$-sequence.
\end{abstract}

\section{Introduction}

The $g$-theorem gives a complete characterization of the $f$-vectors of boundary complexes of simplicial polytopes. It was conjectured by McMullen in 1970 and proved by Billera and Lee [5] (sufficiency) and by Stanley [13] (necessity) in 1980. A major open problem in $f$-vector theory is the $g$-conjecture, which asserts that this characterization holds for all homology spheres. The open part of this conjecture is to show that the $g$-vector of every homology sphere is an $M$-sequence, i.e. it is the $f$-vector of some order ideal of monomials. Based on the fact that homology spheres are doubly Cohen-Macaulay (abbreviated 2-CM) and that the $g$-vector of some other classes of 2-CM complexes is known to be an $M$-sequence (e.g. [14]), Björner and Swartz [14] recently suspected that

Conjecture 1.1 ([14], a weakening of Problem 4.2.) The g-vector of any 2-CM complex is an $M$-sequence.

\section{E. Nevo (ه)}

Institute of Mathematics, The Hebrew University, Jerusalem, Israel

e-mail: eranevo@math.huji.ac.il 
We prove a first step in this direction, namely:

Theorem 1.2 Let $K$ be a $(d-1)$-dimensional 2-CM simplicial complex (over some field) where $d \geq 4$. Then $\left(g_{0}(K), g_{1}(K), g_{2}(K)\right)$ is an $M$-sequence.

This theorem follows from the following theorem, combined with an interpretation of rigidity in terms of the face ring (Stanley-Reisner ring), due (implicitly) to Lee [10].

Theorem 1.3 Let $K$ be a $(d-1)$-dimensional 2-CM simplicial complex (over some field) where $d \geq 3$. Then $K$ has a generically $d$-rigid 1 -skeleton.

Kalai [8] showed that if a simplicial complex $K$ of dimension $\geq 2$ satisfies the following conditions then it satisfies Barnette's lower bound inequalities:

(a) $K$ has a generically $(\operatorname{dim}(K)+1)$-rigid 1 -skeleton.

(b) For each face $F$ of $K$ of codimension $>2$, its link $l k_{K}(F)$ has a generically $\left(\operatorname{dim}\left(l k_{K}(F)\right)+1\right)$-rigid 1-skeleton.

(c) For each face $F$ of $K$ of codimension 2, its link $l k_{K}(F)$ (which is a graph) has at least as many edges as vertices.

Kalai used this observation to prove that Barnette's inequalities hold for a large class of simplicial complexes.

Observe that the link of a vertex in a 2-CM simplicial complex is 2-CM, and that a 2-CM graph is 2-connected. Combining it with Theorem 1.3 and the above result of Kalai we conclude:

Corollary 1.4 Let $K$ be a $(d-1)$-dimensional 2-CM simplicial complex where $d \geq$ 3. For all $0 \leq i \leq d-1 f_{i}(K) \geq f_{i}(n, d)$ where $f_{i}(n, d)$ is the number of $i$-faces in a (equivalently every) stacked d-polytope on $n$ vertices. (Explicitly, $f_{d-1}(n, d)=$ $(d-1) n-(d+1)(d-2)$ and $f_{i}(n, d)=\left(\begin{array}{c}d \\ i\end{array}\right) n-\left(\begin{array}{c}d+1 \\ i+1\end{array}\right)$ i for $\left.1 \leq i \leq d-2.\right)$

Theorem 1.3 is proved by decomposing $K$ into a union of minimal $(d-1)$-cycle complexes (Fogelsanger's notion [6]). Each of these pieces has a generically $d$-rigid 1 -skeleton ([6]), and the decomposition is such that gluing the pieces together results in a complex with a generically $d$-rigid 1 -skeleton. The decomposition is detailed in Theorem 3.4.

This paper is organized as follows: In Sect. 2 we give the necessary background from rigidity theory, explain the connection between rigidity and the face ring, and reduce the results mentioned in the Introduction to Theorem 3.4. In Sect. 3 we give the necessary background on 2-CM complexes, prove Theorem 3.4 and discuss related problems and results.

\section{Rigidity}

The presentation of rigidity here is based mainly on the one in Kalai [8]. 
Let $G=(V, E)$ be a graph. A map $f: V \rightarrow \mathbb{R}^{d}$ is called a $d$-embedding. It is rigid if any small enough perturbation of it which preserves the lengths of the edges is induced by an isometry of $\mathbb{R}^{d}$. Formally, $f$ is called rigid if there exists an $\varepsilon>0$ such that if $g: V \rightarrow \mathbb{R}^{d}$ satisfies $d(f(v), g(v))<\varepsilon$ for every $v \in V$ and $d(g(u), g(w))=$ $d(f(u), f(w))$ for every $\{u, w\} \in E$, then $d(g(u), g(w))=d(f(u), f(w))$ for every $u, w \in V$ (where $d(a, b)$ denotes the Euclidean distance between the points $a$ and $b$ ).

$G$ is called generically $d$-rigid if the set of its rigid $d$-embeddings is open and dense in the topological vector space of all of its $d$-embeddings.

Let $V=[n]$, and let $\operatorname{Rig}(G, f)$ be the $d n \times|E|$ matrix which is defined as follows: for its column corresponding to $\{v<u\} \in E$ put the vector $f(v)-f(u)$ (resp. $f(u)-$ $f(v))$ at the entries of the $d$ rows corresponding to $v$ (resp. $u$ ) and zero otherwise. $G$ is generically $d$-rigid iff $\operatorname{Im}(\operatorname{Rig}(G, f))=\operatorname{Im}\left(\operatorname{Rig}\left(K_{V}, f\right)\right.$ for a generic $f$, where $K_{V}$ is the complete graph on $V . \operatorname{Rig}(G, f)$ is called the rigidity matrix of $G$ (its rank is independent of the generic $f$ that we choose).

Let $G$ be the 1 -skeleton of a $(d-1)$-dimensional simplicial complex $K$. We define $d$ generic degree-one elements in the polynomial ring $A=\mathbb{R}\left[x_{1}, \ldots, x_{n}\right]$ as follows: $\Theta_{i}=\sum_{v \in[n]} f(v)_{i} x_{v}$ where $f(v)_{i}$ is the projection of $f(v)$ on the $i$-th coordinate, $1 \leq i \leq d$. Then the sequence $\Theta=\left(\Theta_{1}, \ldots, \Theta_{d}\right)$ is a linear system of parameters for the face ring $\mathbb{R}[K]=A / I_{K}$ ( $I_{K}$ is the ideal in $A$ generated by the monomials whose support is not an element of $K)$. Let $H(K)=\mathbb{R}[K] /(\Theta)=H(K)_{0} \oplus H(K)_{1} \oplus \cdots$ where $(\Theta)$ is the ideal in $A$ generated by the elements of $\Theta$ and the grading is induced by the degree grading in $A$. Consider the multiplication map $\omega: H(K)_{1} \longrightarrow H(K)_{2}$, $m \rightarrow \omega m$ where $\omega=\sum_{v \in[n]} x_{v}$. Lee [10] proved that

$$
\operatorname{dim}_{\mathbb{R}} \operatorname{Ker}(\operatorname{Rig}(G, f))=\operatorname{dim}_{\mathbb{R}} H(K)_{2}-\operatorname{dim}_{\mathbb{R}} \omega\left(H(K)_{1}\right) .
$$

Assume that $G$ is generically $d$-rigid. Then $\operatorname{dim}_{\mathbb{R}} \operatorname{Ker}(\operatorname{Rig}(G, f))=f_{1}(K)-$ $\operatorname{rank}\left(\operatorname{Rig}\left(K_{V}, f\right)\right)=g_{2}(K)=\operatorname{dim}_{\mathbb{R}} H(K)_{2}-\operatorname{dim}_{\mathbb{R}} H(K)_{1}$. Combining with (1), the map $\omega$ is injective, and hence $\operatorname{dim}_{\mathbb{R}}(H(K) /(\omega))_{i}=g_{i}(K)$ for $i=2$; clearly this holds for $i=0,1$ as well. Hence $\left(g_{o}(K), g_{1}(K), g_{2}(K)\right)$ is an $M$-sequence. We conclude that Theorem 1.3 implies Theorem 1.2, via the following algebraic result:

Theorem 2.1 Let $K$ be a $(d-1)$-dimensional 2-CM simplicial complex (over some field) where $d \geq 3$. Then the multiplication map $\omega: H(K)_{1} \longrightarrow H(K)_{2}$ is injective.

In order to prove Theorem 1.3, we need the concept of minimal cycle complexes, introduced by Fogelsanger [6]. We summarize his theory below.

Fix a field $k$ (or more generally, any Abelian group) and consider the formal chain complex on a ground set $[n], C=(\bigoplus\{k T: T \subseteq[n]\}, \partial)$, where $\partial(1 T)=$ $\sum_{t \in T} \operatorname{sign}(t, T) T \backslash\{t\}$ and $\operatorname{sign}(t, T)=(-1)^{|\{s \in T: s<t\}|}$. Define subchain, minimal $d-$ cycle and minimal $d$-cycle complex as follows: $c^{\prime}=\sum\left\{b_{T} T: T \subseteq[n],|T|=d+1\right\}$ is a subchain of a $d$-chain $c=\sum\left\{a_{T} T: T \subseteq[n],|T|=d+1\right\}$ iff for every such $T$, $b_{T}=a_{T}$ or $b_{T}=0$. A $d$-chain $c$ is a $d$-cycle if $\partial(c)=0$, and is a minimal $d$-cycle if its only subchains which are cycles are $c$ and 0 . A simplicial complex $K$ which is spanned by the support of a minimal $d$-cycle is called a minimal $d$-cycle complex (over $k$ ), i.e. $K=\left\{S: \exists T S \subseteq T, a_{T} \neq 0\right\}$ for some minimal $d$-cycle $c$ as above. For example, triangulations of connected manifolds without boundary are minimal cycle complexes-fix $k=\mathbb{Z}_{2}$ and let the cycle be the sum of all facets. 
The following is the main result in Fogelsanger's thesis.

Theorem 2.2 (Fogelsanger [6]) For $d \geq 3$, every minimal $(d-1)$-cycle complex has a generically d-rigid 1-skeleton.

We will need the following gluing lemma, due of Asimov and Roth, who introduced the concept of generic rigidity of graphs [1].

Theorem 2.3 (Asimov and Roth [2]) Let $G_{1}$ and $G_{2}$ be generically d-rigid graphs. If $G_{1} \cap G_{2}$ contains at least $d$ vertices, then $G_{1} \cup G_{2}$ is generically d-rigid.

Now we are ready to conclude Theorem 1.3 from the decomposition theorem, Theorem 3.4.

Proof of Theorem 1.3 Consider a decomposition sequence of $K$ as guaranteed by Theorem 3.4, $K=\bigcup_{i=1}^{m} S_{i}$. By Theorem 2.2 each $S_{i}$ has a generically $d$-rigid 1 skeleton. By Theorem 2.3 for all $2 \leq i \leq m \bigcup_{j=1}^{i} S_{j}$ has a generically $d$-rigid 1skeleton, in particular $K$ has a generically $d$-rigid 1-skeleton $(i=m)$.

Remark One can verify that Theorems 2.2 and 2.3, and hence also Theorem 1.3, continue to hold when replacing "generically $d$-rigid" by the notion " $d$-hypperconnected", introduced by Kalai [7]. Both of these assertions have an interpretation in terms of algebraic shifting, introduced by Kalai (see e.g. his survey [9]), namely: for both the exterior and symmetric shifting operators over the field $\mathbb{R}$, denoted by $\Delta,\{d, n\} \in \Delta(K)$. The existence of this edge in the shifted complex implies the nonnegativity of $g_{2}(K)$.

\section{Decomposing a 2-CM Complex}

Definition 3.1 A simplicial complex $K$ is $2-C M$ (over a fixed field $k$ ) if it is CohenMacaulay and for every vertex $v \in K, K-v$ is Cohen-Macaulay of the same dimension as $K$.

Here $K-v$ is the simplicial complex $\{T \in K: v \notin T\}$. By a theorem of Reisner [11], a simplicial complex $L$ is Cohen-Macaulay iff it is pure and for every face $T \in L$ (including the empty set) and every $i<\operatorname{dim}\left(l k_{L}(T), \tilde{H}_{i}\left(l k_{L}(T) ; k\right)=0\right.$ where $l k_{L}(T)=\{S \in L: T \cap S=\emptyset, T \cup S \in L\}$ and $\tilde{H}_{i}(M ; k)$ is the reduced $i$-th homology of $M$ over $k$. The proof of Theorem 3.4 is by induction on $\operatorname{dim}(K)$. Let us first consider the case where $K$ is 1 -dimensional.

A (simple finite) graph is 2-connected if after a deletion of any vertex from it, the remaining graph is connected and nontrivial (i.e. is not a single vertex nor empty). Note that a graph is 2-CM iff it is 2-connected.

Lemma 3.2 A graph $G$ is 2-connected iff there exists a decomposition $G=\bigcup_{i=1}^{m} C_{i}$ such that each $C_{i}$ is a simple cycle and for every $1<i \leq m, C_{i} \cap\left(\bigcup_{j<i} C_{j}\right)$ contains an edge. 
Moreover, for each $i_{0} \in[m]$ the $C_{i}$ 's can be reordered by a permutation $\sigma:[m] \rightarrow$ $[m]$ such that $\sigma^{-1}(1)=i_{0}$ and for every $i>1, C_{\sigma^{-1}(i)} \cap\left(\bigcup_{j<i} C_{\sigma^{-1}(j)}\right)$ contains an edge.

Proof Whitney [15] showed that a graph $G$ is 2-connected iff it has an open ear decomposition, i.e. there exists a decomposition $G=\bigcup_{i=0}^{m} P_{i}$ such that each $P_{i}$ is a simple open path, $P_{0}$ is an edge, $P_{0} \cup P_{1}$ is a simple cycle and for every $1<i \leq m$ $P_{i} \cap\left(\bigcup_{j<i} P_{j}\right)$ equals the 2 end vertices of $P_{i}$.

Assume that $G$ is 2-connected and consider an open ear decomposition as above. Let $C_{1}=P_{0} \cup P_{1}$. For $i>1$ choose a simple path $\tilde{P}_{i}$ in $\bigcup_{j<i} P_{j}$ that connects the 2 end vertices of $P_{i}$, and let $C_{i}=P_{i} \cup \tilde{P}_{i} .\left(C_{1}, \ldots, C_{m}\right)$ is the desired decomposition sequence of $G$.

Let $C$ be the graph whose vertices are the $C_{i}$ 's and two of them are neighbors iff they have an edge in common. Thus, $C$ is connected, and hence the 'Moreover' part of the Lemma is proved.

The other implication, that such a decomposition implies 2-connectivity, will not be used in the sequel, and its proof is omitted.

For the induction step we need the following cone lemma. For $v$ a vertex not in the support of a $(d-1)$-chain $c$, let $v * c$ denote the following $d$-chain: if $c=\sum\left\{a_{T} T\right.$ : $v \notin T \subseteq[n],|T|=d\}$ where $a_{T} \in k$ for all $T$, then $v * c=\sum\left\{\operatorname{sign}(v, T) a_{T} T \cup\{v\}\right.$ : $v \notin T \subseteq[n],|T|=d\}$ where $\operatorname{sign}(v, T)=(-1)^{|\{t \in T: t<v\}|}$.

Lemma 3.3 Let $s$ be a minimal $(d-1)$-cycle and let $c$ be a minimal $d$-chain such that $\partial(c)=s$, i.e. $c$ has no proper subchain $c^{\prime}$ such that $\partial\left(c^{\prime}\right)=s$. For $v$ a vertex not in any face in $\operatorname{supp}(c)$, the support of $c$, define $\tilde{s}=c-v * s$. Then $\tilde{s}$ is a minimal d-cycle.

Proof $\partial(\tilde{s})=\partial(c)-\partial(v * s)=s-(s-v * \partial(s))=0$ hence $\tilde{s}$ is a $d$-cycle. To show that it is minimal, let $\hat{s}$ be a subchain of $\tilde{s}$ such that $\partial(\hat{s})=0$. Note that $\operatorname{supp}(c) \cap$ $\operatorname{supp}(v * s)=\emptyset$.

Case 1: $v$ is contained in a face in $\operatorname{supp}(\hat{s})$. By the minimality of $s, \operatorname{supp}(v * s) \subseteq$ $\operatorname{supp}(\hat{s})$. Thus, by the minimality of $c$ also $\operatorname{supp}(c) \subseteq \operatorname{supp}(\hat{s})$ and hence $\hat{s}=\tilde{s}$.

Case 2: $v$ is not contained in any face in $\operatorname{supp}(\hat{s})$. Thus, $\operatorname{supp}(\hat{s}) \subseteq \operatorname{supp}(c)$. As $\partial(\hat{s})=$ 0 then $\partial(c-\hat{s})=s$. The minimality of $c$ implies $\hat{s}=0$.

Theorem 3.4 Let $K$ be a d-dimensional 2-CM simplicial complex over a field $k$ $(d \geq 1)$. Then there exists a decomposition $K=\bigcup_{i=1}^{m} S_{i}$ such that each $S_{i}$ is a minimal d-cycle complex over $k$ and for every $i>1, S_{i} \cap\left(\bigcup_{j<i} S_{j}\right)$ contains a $d$-face.

Moreover, for each $i_{0} \in[\mathrm{m}]$ the $S_{i}$ 's can be reordered by a permutation $\sigma:[\mathrm{m}] \rightarrow$ $[m]$ such that $\sigma^{-1}(1)=i_{0}$ and for every $i>1, S_{\sigma^{-1}(i)} \cap\left(\bigcup_{j<i} S_{\sigma^{-1}(j)}\right)$ contains $a$ d-face.

Proof The proof is by induction on $d$. For $d=1$, by Lemma $3.2 K=\bigcup_{i=1}^{m(K)} C_{i}$ such that each $C_{i}$ is a simple cycle and for every $i>1 C_{i} \cap\left(\bigcup_{j<i} C_{j}\right)$ contains an edge. Define $s_{i}=\sum\left\{\operatorname{sign}_{e}(i) e: e \in\left(C_{i}\right)_{1}\right\}$, then $s_{i}$ is a minimal 1-cycle (orient the edges 
properly: $\operatorname{sign}_{e}(i)$ equals 1 or -1 accordingly) whose support spans the simplicial complex $C_{i}$. Moreover, by Lemma 3.2 each $C_{i_{0}}, i_{0} \in[m(K)]$, can be chosen to be the first in such a decomposition sequence.

For $d>1$, note that the link of every vertex in a 2-CM simplicial complex is 2-CM. For a vertex $v \in K$, as $l k_{K}(v)$ is 2-CM then by the induction hypothesis $l k_{K}(v)=$ $\bigcup_{i=1}^{m(v)} C_{i}$ such that each $C_{i}$ is a minimal $(d-1)$-cycle complex and for every $i>1$ $C_{i} \cap\left(\bigcup_{j<i} C_{j}\right)$ contains a $(d-1)$-face. Let $s_{i}$ be a minimal $(d-1)$-cycle whose support spans $C_{i}$. As $K-v$ is $\mathrm{CM}$ of dimension $d, \tilde{H}_{d-1}(K-v ; k)=0$. Hence there exists a $d$-chain $c$ such that $\partial(c)=s_{i}$ and $\operatorname{supp}(c) \subseteq K-v$.

Take $c_{i}$ to be such a chain with a support of minimal cardinality. By Lemma 3.3, $\tilde{s}_{i}=c_{i}-v * s_{i}$ is a minimal $d$-cycle. Let $S_{i}(v)$ by the simplicial complex spanned by $\operatorname{supp}\left(\tilde{s}_{i}\right)$; it is a minimal $d$-cycle complex. By the induction hypothesis, for every $i>$ $1 S_{i}(v) \cap\left(\bigcup_{j<i} S_{j}(v)\right)$ contains a $d$-face (containing $\left.v\right)$. Thus, $K(v):=\bigcup_{j=1}^{m(v)} S_{j}(v)$ has the desired decomposition for every $v \in K . K=\bigcup_{v \in \operatorname{Ver}(K)} K(v)$ as $s t_{K}(v) \subseteq$ $K(v)$ for every $v$, where $s t_{K}(v)=\{T \in K: T \cup\{v\} \in K\}$.

Let $v$ be any vertex of $K$. Since the 1-skeleton of $K$ is connected, we can order the vertices of $K$ such that $v_{1}=v$ and for every $i>1 v_{i}$ is a neighbor of some $v_{j}$ where $1 \leq j<i$. Let $v_{l(i)}$ be such a neighbor of $v_{i}$. By the induction hypothesis we can order the $S_{j}\left(v_{i}\right)$ 's such that $S_{1}\left(v_{i}\right)$ will contain $v_{l(i)}$, and hence, as $K$ is pure, will contain a $d$-face which appears in $K\left(v_{l(i)}\right)$ (this face contains the edge $\left.\left\{v_{i}, v_{l(i)}\right\}\right)$. The resulting decomposition sequence $\left(S_{1}\left(v_{1}\right), \ldots, S_{m\left(v_{1}\right)}\left(v_{1}\right), S_{1}\left(v_{2}\right), \ldots, S_{m\left(v_{n}\right)}\left(v_{n}\right)\right)$ is as desired.

Moreover, every $S_{j}\left(v_{i_{0}}\right)$ where $i_{0} \in[n]$ and $j \in\left[m\left(v_{i_{0}}\right)\right]$ can be chosen to be the first in such a decomposition sequence. Indeed, by the induction hypothesis $S_{j}\left(v_{i_{0}}\right)$ can be the first in the decomposition sequence of $K\left(v_{i_{0}}\right)$, and as mentioned before, the connectivity of the 1-skeleton of $K$ guarantees that each such prefix $\left(S_{1}\left(v_{i_{0}}\right), \ldots, S_{m\left(v_{i_{0}}\right)}\left(v_{i_{0}}\right)\right)$ can be completed to a decomposition sequence of $K$ on the same $S_{j}\left(v_{i}\right)$ 's.

Theorem 1.3 follows also from the following corollary combined with Theorem 2.2.

Corollary 3.5 Let $K$ be a d-dimensional 2-CM simplicial complex over a field $k$ $(d \geq 1)$. Then $K$ is a minimal cycle complex over the Abelian group $\tilde{k}=k\left(x_{1}, x_{2}, \ldots\right)$ whose elements are finite linear combinations of the (variables) $x_{i}$ 's with coefficients in $k$.

Proof Consider a decomposition $K=\bigcup_{i=1}^{m} S_{i}$ as guaranteed by Theorem 3.4, where $S_{i}=\overline{\operatorname{supp}\left(c_{i}\right)}$ (the closure w.r.t. inclusion of $\operatorname{supp}\left(c_{i}\right)$ ) for some minimal $d$-cycle $c_{i}$ over $k$. Define $\tilde{c}_{i}=x_{i} c_{i}$, thus $\tilde{c}_{i}$ is a minimal cycle over $\tilde{k}$. Define $\tilde{c}=\sum_{i=1}^{m} \tilde{c}_{i}$. Clearly $\tilde{c}$ is a cycle over $\tilde{k}$ whose support spans $K$. It remains to show that $\tilde{c}$ is minimal. Let $\tilde{c}^{\prime}$ be a subchain of $\tilde{c}$ which is a cycle, $\tilde{c}^{\prime} \neq \tilde{c}$. We need to show that $\tilde{c}^{\prime}=0$. Denote by $\tilde{\alpha}_{T}\left(\tilde{\alpha}_{T}^{\prime}\right)$ the coefficient of the set $T$ in $\tilde{c}\left(\tilde{c}^{\prime}\right)$ and by $\tilde{\alpha}_{T}(i)$ the coefficient of the set $T$ in $\tilde{c}_{i}$. If $\tilde{\alpha}_{T}^{\prime}=0$ then for every $i$ such that $\tilde{\alpha}_{T}(i) \neq 0$, the minimality of $\tilde{c}_{i}$ implies that $\tilde{\alpha}_{F}^{\prime}=0$ whenever $\tilde{\alpha}_{F}(i) \neq 0$. By assumption, there exists a set $T_{0}$ such that $\tilde{\alpha}_{T_{0}}^{\prime}=0 \neq \tilde{\alpha}_{T_{0}}$. In particular, there exists an index $i_{0}$ such 
that $\tilde{\alpha}_{T_{0}}\left(i_{0}\right) \neq 0$, hence $\tilde{\alpha}_{F}^{\prime}=0$ whenever $\tilde{\alpha}_{F}\left(i_{0}\right) \neq 0$. As $S_{i_{0}} \cap\left(\bigcup_{j<i_{0}} S_{j}\right)$ contains a $d$-face in case $i_{0}>1$, repeated application of the above argument implies $\tilde{\alpha}_{F}^{\prime}=0$ whenever $\tilde{\alpha}_{F}(1) \neq 0$. Repeated application of the fact that $S_{i} \cap\left(\bigcup_{j<i} S_{j}\right)$ contains a $d$-face for $i=2,3, \ldots$ and of the above argument shows that $\tilde{\alpha}_{F}^{\prime}=0$ whenever $\tilde{\alpha}_{F}(i) \neq 0$ for some $1 \leq i \leq m$, i.e. $\tilde{c}^{\prime}=0$.

A pure simplicial complex has a nowhere zero flow if there is an assignment of integer non-zero wights to all of its facets which forms a $\mathbb{Z}$-cycle. This generalizes the definition of a nowhere zero flow for graphs (e.g. [12] for a survey).

Corollary 3.6 Let $K$ be a d-dimensional 2-CM simplicial complex over $\mathbb{Q}(d \geq 1)$. Then $K$ has a nowhere zero flow.

Proof Consider a decomposition $K=\bigcup_{i=1}^{m} S_{i}$ as guaranteed by Theorem 3.4. Multiplying by a common denominator, we may assume that each $S_{i}=\overline{\operatorname{supp}\left(c_{i}\right)}$ for some minimal $d$-cycle $c_{i}$ over $\mathbb{Z}$ (instead of just over $\mathbb{Q}$ ). Let $N$ be the maximal $|\alpha|$ over all nonzero coefficients $\alpha$ of the $c_{i}$ 's, $1 \leq i \leq m$. Let $\tilde{c}=\sum_{i=1}^{m}\left(N^{m}\right)^{i} c_{i} . \tilde{c}$ is a nowhere zero flow for $K$; we omit the details.

Problem 3.7 Can the $S_{i}$ 's in Theorem 3.4 be taken to be homology spheres?

Yhonatan Iron and I proved (unpublished) the following lemma:

Lemma 3.8 Let $K, L$ and $K \cap L$ be simplicial complexes of the same dimension $d-1$. Assume that $K$ and $L$ are weak-Lefschetz, i.e. that multiplication by a generic degree-one element $g$ in $H=H(K), H(L), g: H_{i-1} \rightarrow H_{i}$, is injective for all $i \leq$ $\lfloor d / 2\rfloor$. If $K \cap L$ is $C M$ then $K \cup L$ is weak-Lefschetz.

In view of this lemma, if the intersections $S_{i} \cap\left(\bigcup_{j<i} S_{j}\right)$ in Theorem 3.4 can be taken to be CM, and the $S_{i}$ 's can be taken to be homology spheres, then Conjecture 1.1 would be reduced to the long standing $g$-conjecture for homology spheres. Can the intersections be guaranteed to be CM?

Acknowledgements I would like to thank my adviser Gil Kalai, Anders Björner and Ed Swartz for helpful discussions. This research was done during the author's stay at Institut Mittag-Leffler, supported by the ACE network.

\section{References}

1. Asimov, L., Roth, B.: The rigidity of graphs. Trans. Am. Math. Soc. 245, 279-289 (1978)

2. Asimov, L., Roth, B.: The rigidity of graphs: part II. J. Math. Anal. Appl. 68, 171-190 (1979)

3. Barnette, D.: The minimum number of vertices of a simple polytope. Isr. J. Math. 10, 121-125 (1971)

4. Barnette, D.: A proof of the lower bound conjecture for convex polytopes. Pac. J. Math. 46, 349-354 (1973)

5. Billera, L.G., Lee, C.W.: A proof of the sufficiency of McMullen conditions for $f$-vectors of simplicial convex polytopes. J. Comb. Theory Ser. A 31, 237-255 (1981)

6. Fogelsanger, A.: The generic rigidity of minimal cycles. PhD dissertation, Cornell University (1988). Also at http://www.people.cornell.edu/pages/alf6/rigidity.htm 
7. Kalai, G.: Hyperconnectivity of graphs. Graphs Comb. 1, 65-79 (1985)

8. Kalai, G.: Rigidity and the lower bound theorem. Invent. Math. 88, 125-151 (1987)

9. Kalai, G.: Algebraic shifting. Adv. Stud. Pure Math. 33, 121-163 (2002)

10. Lee, K.W.: Generalized stress and motion. In: Briztriczky, T., et al. (eds.) Polytopes: Abstract, Convex and Computational, pp. 249-271. Kluwer Academic, Dordrecht (1995)

11. Reisner, G.: Cohen-Macaulay quotients of polynomial rings. Adv. Math. 21, 30-49 (1976)

12. Seymour, P.D.: Nowhere-zero flows. In: Graham, R., et al. (eds.) Handbook of Combinatorics, pp. 289-299. Elsevier, Amsterdam (1995)

13. Stanley, R.P.: The number of faces of simplicial convex polytopes. Adv. Math. 35, 236-238 (1980)

14. Swartz, E.: g-elements, finite buildings and higher Cohen-Macaulay connectivity. J. Comb. Theory Ser. A 113, 1305-1320 (2006)

15. Whitney, H.: Non-separable and planar graphs. Trans. Am. Math. Soc. 34, 339-362 (1932) 